\title{
Actualización de la depresión postictus: nuevos retos en pacientes con ictus minor 0 ataque isquémico transitorio
}

\author{
Anna Carnés-Vendrell, Joan Deus-Yela, Jessica Molina-Seguin, Josep Pifarré-Paradero, Francisco Purroy
}

Unidad de Investigación en Resonancia Magnética; Hospital del Mar; Barcelona (J. Deus-Yela). Departamento de Psicología Clínica y de la Salud; Facultad de Psicología; Universitat Autònoma de Barcelona; Barcelona (J. Deus-Yela). Institut de Recerca Biomèdica de Lleida; Grupo de Neurociencias Clínicas; Universitat de Lleida (A. CarnésVendrell, J. Molina-Seguin, J. PifarréParadero, F. Purroy). Unidad de Ictus; Hospital Universitari Arnau

de Vilanova; Lleida, España

(J. Molina-Seguin, F. Purroy)

Correspondencia:

Dr. Francisco Purroy García. Unidad de Ictus. Hospital Universitar Arnau de Vilanova. Alcalde Rovira Roure, 80. E-25198 Lleida.

E-mail:

fpurroygarcia@gmail.com

Aceptado tras revisión externa: 04.04.16.

Cómo citar este artículo Carnés-Vendrell A, Deus-Yela J, Molina-Seguin J, Pifarré-Paredero J

Purroy F. Actualización de la depresión postictus: nuevos retos en pacientes con ictus minor o ataque isquémico transitorio. Rev Neurol 2016; 62: 460-7.

C 2016 Revista de Neurología

Introducción. El ictus es una de las principales causas de discapacidad en la población adulta. El desarrollo de síntomas depresivos es la complicación afectiva más frecuente. Hasta ahora, en la mayoría de los estudios sobre depresión postictus se ha excluido a los pacientes que han sufrido un ictus minor o un ataque isquémico transitorio (AIT), si bien es un subgrupo igualmente vulnerable a esta enfermedad.

Objetivo. Revisar los estudios publicados de depresión postictus para dilucidar los aspectos que ya se han demostrado ampliamente y los que necesitan mayor evidencia.

Desarrollo. La depresión postictus es frecuente tanto en los pacientes con ictus establecido como en los pacientes con ictus minor o AIT. Aunque existen discrepancias en la definición utilizada, aproximadamente uno de cada tres pacientes desarrollará esta complicación. Se han identificado factores de riesgo de depresión postictus con un amplio respaldo científico (sexo femenino, antecedentes de depresión u otros trastornos psiquiátricos, gravedad del ictus y afectación funcional) y otros sin él (calidad de vida, deterioro cognitivo y biomarcadores de neuroimagen). Las principales limitaciones metodológicas halladas son la confusión entre depresión postictus y sintomatología depresiva, la variabilidad en las escalas de evaluación usadas y la variabilidad en el momento temporal de la evaluación del estado de ánimo. Hasta ahora son muy pocos los estudios en el ictus minor o el AIT.

Conclusiones. Se necesitan nuevos estudios con mejor diseño que ayuden a establecer el riesgo de depresión postictus a diferentes tiempos tras el ictus, el ictus minor o el AIT, y establecer la importancia de los factores descritos previamente.

Palabras clave. Ataque isquémico transitorio. Depresión postictus. Ictus minor. Neuropsicología. Pronóstico. Revisión.

\section{Introducción}

El ictus constituye una verdadera pandemia de la sociedad occidental. Una de cada diez muertes se debe a esta enfermedad. Además, se considera la principal causa de discapacidad en la población adulta y la segunda causa de demencia [1]. Durante las últimas décadas se han destinado enormes y esperanzadores esfuerzos en el tratamiento de la fase aguda del ictus isquémico para cambiar la historia natural de la enfermedad. La aprobación del tratamiento fibrinolítico dentro de las primeras cuatro horas y media del ictus, y la demostración de la seguridad y efectividad de las terapias mecánicas combinadas han revolucionado la actitud terapéutica en estos pacientes [2-5]. Sin embargo, más allá de la fase aguda existen ciertos aspectos que merecen también atención y que mejoran el pronóstico y la calidad de vida de estos pacientes, como el tratamiento rehabilitador, el tratamiento de prevención secundaria y el estado emocional.

En los últimos años ha crecido el interés científico por esta última faceta. El desarrollo de síntomas depresivos es la complicación afectiva más frecuente después del ictus. La mayoría de los estudios que se han llevado a cabo sobre la depresión postictus se ha centrado en determinar su prevalencia y los factores de riesgo asociados. Las discrepancias en el diseño de los estudios han hecho que el rango de prevalencia notificado varíe enormemente. No obstante, revisiones sistemáticas publicadas al respecto comunican una prevalencia global del 33\% [6]. Estos datos varían en función de la población estudiada y del momento de realizar la evaluación. Así, en estudios de base poblacional, la prevalencia oscila del $20 \%$ al $40 \%$ y asciende a más del $40 \%$ en pacientes hospitalizados [7]. La prevalencia varía en función del momento de su determinación tras el ictus. Según una revisión de Carod-Artal, la prevalencia en las primeras dos semanas va del $6 \%$ al $40 \%$, a los tres meses llega al 50\%, es del $20 \%$ al $50 \%$ al cabo de un año, y disminuye al 20\% a los dos años [8]. Finalmente, si analizamos los síntomas depresivos postictus y no sólo el diagnóstico de la depresión, la prevalencia puede aumentar hasta el $78 \%$ de los pacientes [9]. 
A continuación se expone una actualización sobre los estudios publicados hasta la fecha sobre la depresión postictus con el objetivo de clarificar los aspectos que ya han sido ampliamente demostrados y de poner luz sobre los que requieren mayores esfuerzos en investigación para seguir avanzando en la comprensión y tratamiento de esta enfermedad. A lo largo de la revisión pondremos el foco de atención en un subgrupo de ictus que también se encuentra bajo la amenaza de esta complicación y que a menudo no ha recibido tanta atención, como los pacientes que han sufrido un ictus minor o un ataque isquémico transitorio (AIT).

\section{Metodología}

Se utilizó la base de datos PubMed, utilizando cómo filtro las palabras clave 'stroke', 'minor stroke, 'transient ischemic attack', 'poststroke depression', 'depressive disorder', 'apathy', 'epidemiology', 'neuropsychology', 'cognition disorders', 'risk factors', 'predictors', 'consequences', 'suicide', 'vascular depression' y 'dysexecutive depression'. Se incluyeron los artículos originales publicados en inglés, castellano o portugués que estudiaban la prevalencia de la depresión postictus, los factores de riesgo asociados a la depresión postictus, así como los que analizaban la relación entre el ictus, la depresión postictus y el rendimiento cognitivo, y finalmente los estudios que analizan las consecuencias de la depresión postictus para los pacientes que la sufren. De un total de 225 artículos encontrados y analizados, se excluyeron los publicados con anterioridad al año 2000 (con tres excepciones, dada su contribución científica), y los casos clínicos y estudios realizados en animales. De esta forma, en total se incluyeron 62 artículos.

\section{Variabilidad en los estudios de depresión postictus}

Antes de analizar los resultados encontrados con esta revisión, es importante señalar cuáles han sido a nuestro entender las posibles limitaciones que han conllevado esta enorme variabilidad en los estudios de depresión postictus. Las discrepancias en la prevalencia de la depresión postictus según el momento de detección tras el ictus y el tipo de cohorte estudiada se pueden justificar por diversas limitaciones en la metodología utilizada en los diferentes estudios. Las posibles limitaciones se enumeran a continuación:
- La propia definición de la depresión postictus, es decir, los criterios diagnósticos utilizados. Si bien en algunos artículos se defiende la utilización de los criterios del Manual diagnóstico y estadístico de los trastornos mentales (DSM, en sus versiones III, IV-TR o 5 en función del año), otros muchos se basan en la puntuación de las escalas clínicas de evaluación para poder llegar al diagnóstico. De esta forma, se puede dar el caso de que sujetos con sintomatología depresiva postictus sean considerados como sujetos con depresión postictus, sin cumplir estrictamente con los criterios diagnósticos propuestos en el DSM. Este problema metodológico ya fue avisado por Paolucci et al en su estudio observacional multicéntrico de depresión postictus en Italia. Indicaron que en publicaciones previas se estaba considerando como población con depresión postictus a pacientes tanto con distimia como con trastorno depresivo mayor y trastorno adaptativo [10], y todos ellos eran trastornos con criterios diagnósticos diferentes.

- La anterior limitación enlaza con la dificultad para establecer un correcto diagnóstico diferencial entre los síntomas depresivos somáticos con los síntomas derivados del propio ictus, como pueden ser el insomnio, la pérdida de apetito o los síntomas vegetativos de ansiedad, entre otros [11]. Resulta en ocasiones un verdadero reto distinguir si los síntomas que sufre el paciente forman parte del cuadro depresivo o son alteraciones del sistema autonómico. Por otro lado, la apatía ha sido motivo de numerosas publicaciones, y se puede encontrar de forma independiente al trastorno depresivo o como un síntoma más de la depresión. Algunos autores informan de una prevalencia de la apatía postictus como síndrome independiente de la depresión postictus del 10$55 \%$, lo que hace importante su diagnóstico diferencial [12-17]. Por último, se han descrito otras alteraciones tras el ictus que deben analizarse con cautela para no confundirlas con síntomas depresivos. Destacan en este sentido la labilidad emocional, la aprosodia afectiva o la fatiga [15].

- Otra limitación es la gran variabilidad en las escalas de evaluación usadas. Encontramos varios estudios que comparan la validez de distintas escalas clínicas con resultados dispares. Tanto el inventario de depresión de Beck como la escala de depresión de Montgomery-Åsberg han sido apoyados como instrumentos psicométricos para la detección de depresión postictus [18-22]. Meader et al han realizado un metaanálisis sobre los instrumentos de cribado disponibles para la de- 
presión postictus, y concluyen que ningún instrumento es válido en sí mismo para realizar el diagnóstico, ya que éste debe hacerse mediante entrevista clínica [23].

- Respecto al momento temporal de la evaluación del estado de ánimo del sujeto, parece no existir un consenso en cuándo determinar si un paciente sufre una depresión postictus. En la bibliografía hay una gran variabilidad que va desde la fase aguda a meses e incluso años después del ictus. Esta variabilidad se traduce en una dispersión de datos sobre la prevalencia de depresión postictus.

- Finalmente, los criterios utilizados para seleccionar las muestras de estudio. Muchos estudios son muy estrictos en la exclusión de pacientes. Así, la gran mayoría de los artículos publicados en relación con la depresión postictus describen en el apartado de metodología la exclusión de pacientes con ictus minor o AIT, lo que dificulta poder determinar la prevalencia de depresión en estos sujetos, analizar cómo evolucionan o determinar cuáles son los factores de riesgo en este subgrupo de población. Hoy en día, pese a la transitoriedad de los síntomas o su práctica resolución, hay un consenso en considerar estos pacientes no sólo vulnerables por lo que se refiere al riesgo de recurrencia, sino, como demuestran estudios recientes, también para la depresión postictus o los síntomas depresivos [24-26]. Otros artículos excluyen también de sus muestras a los sujetos con afasia, con deterioro cognitivo grave e incluso con antecedentes psiquiátricos personales, eliminando por tanto a los sujetos con mayores probabilidades de desarrollar depresión postictus porque se ha demostrado que los antecedentes psiquiátricos personales son un factor de riesgo principal para el desarrollo de depresión postictus.

\section{¿Diferentes nomenclaturas para un mismo cuadro? De la depresión vascular a la depresión postictus y la depresión disejecutiva}

En la bibliografía se diferencian tres conceptos relacionados con la alteración emocional tras el ictus: la depresión vascular, la depresión postictus y la depresión disejecutiva, aunque los tres podrían ser nomenclaturas de un mismo cuadro clínico. Los síntomas cardinales para el diagnóstico de la depresión vascular son: evidencia clínica o de exploraciones complementarias de enfermedad vascular o factores de riesgo vasculares, junto con el inicio de la depresión en personas mayores de 65 años o el cambio en el curso de la depresión después de la aparición de la enfermedad vascular, y el desarrollo de episodios depresivos más frecuentes y persistentes. A estas características principales se añaden otras características secundarias que apoyan el diagnóstico: presencia de deterioro cognitivo consistente en déficit en las funciones ejecutivas, aunque no exclusivo, enlentecimiento psicomotor, ideación depresiva limitada (escasa presencia de síntomas como la desesperanza), escasa conciencia de enfermedad, discapacidad física y ausencia de antecedentes familiares de trastornos del estado de ánimo [27]. Más tarde, Lockwood et al enfatizaron que los déficits en las funciones ejecutivas son una característica central en la depresión geriátrica [28], y Alexopoulos acuñó el término 'síndrome de depresión-disfunción ejecutiva en la vejez', que más tarde se conocería popularmente como depresión disejecutiva [29]. La depresión disejecutiva tiene, por tanto, una entidad propia dentro de las depresiones vasculares. Sus síntomas depresivos cursan con un peor funcionamiento psicosocial y peor afrontamiento en las actividades de la vida diaria. Por ello, se puede considerar una forma más grave de depresión vascular.

En cambio, la depresión postictus hace referencia a un período importante y persistente de estado de ánimo deprimido o una disminución notable del interés o placer por todas o casi todas las actividades tras un ictus. Para ser exactos, el DSM-5 categoriza la depresión postictus como un trastorno depresivo debido a otra afección médica, en este caso el ictus, y debe especificarse si se manifiesta con características depresivas, con episodio del tipo de depresión mayor o con características mixtas [30]. Los síntomas de la depresión postictus, aunque pueden afectar a las mismas áreas que el trastorno depresivo mayor primario (afectiva, somática y cognitiva), tienen unas características fenomenológicas diferentes que por cuestiones de espacio no abordaremos en esta revisión. Se entiende, pues, que la depresión postictus puede considerarse una forma de depresión vascular al ser secundaria a la patología vascular. En consecuencia, se podría entender que tanto la depresión disejecutiva como la depresión postictus son subtipos de depresión vascular, dentro de un continuo de gravedad y afectación.

\section{Factores de riesgo y predictores de la depresión postictus}

No sólo es de interés concretar la importancia del problema, sino que también es valioso poder determinar los factores de riesgo y predictores de depre- 
Tabla. Factores de riesgo y predictores de depresión postictus con respecto al consenso en la literatura científica.

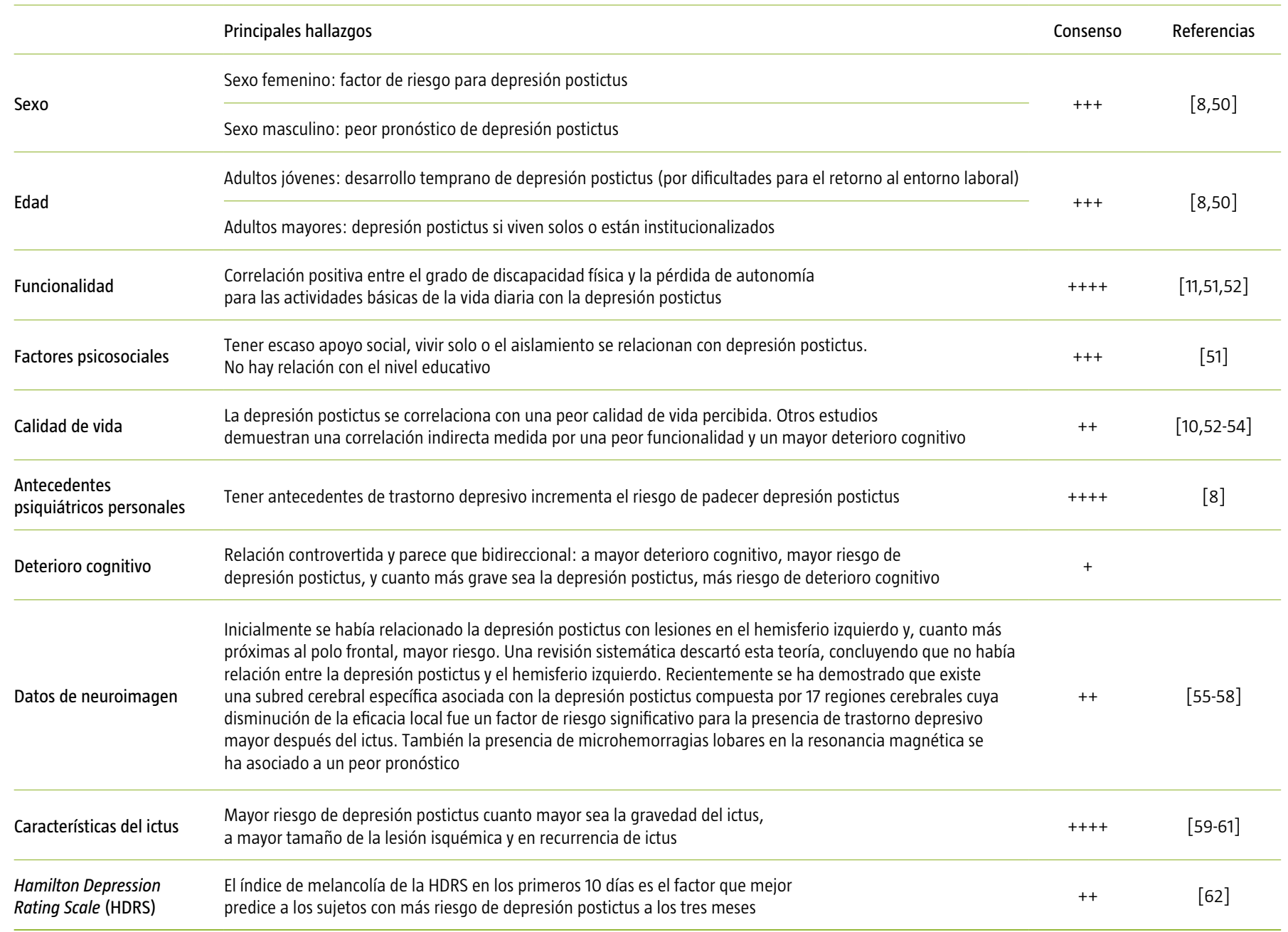

sión postictus para poder identificar los pacientes más frágiles. En la tabla se presentan los principales hallazgos hasta el momento con su respectiva valoración del consenso existente en la bibliografía.

Resumiendo, el sexo femenino, la presencia de antecedentes de depresión $\mathrm{u}$ otros trastornos psiquiátricos personales, la gravedad del ictus, las consecuencias funcionales (discapacidad física o pérdida de autonomía para las actividades básicas de la vida diaria), vivir solo o tener escaso apoyo social después del ictus se han descrito como factores de riesgo para la depresión postictus [31]. A pesar de la amplia gama de variables estudiadas en las últimas dos décadas, persiste la incertidumbre acerca de los factores de riesgo específicos para depresión postictus, ya que los datos disponibles provienen mayoritariamente de estudios de tamaño y, a veces, diseño insuficiente para permitir extrapolar los resultados de unas cohortes a otras.

\section{Depresión postictus y rendimiento cognitivo}

Existe tradición en cuanto a relacionar el desarrollo de la depresión postictus y el rendimiento cognitivo. De hecho, el deterioro cognitivo tras haber sufrido un ictus es frecuente y se estima entre el 35,2\% $y$ el $87 \%$ de los pacientes [32], y hasta un $25-31 \%$ puede llegar a sufrir demencia postictus [33]. Como en anteriores apartados, la revisión de la bibliografía existente específica sobre esta cuestión muestra diversidad de resultados y heterogeneidad metodo- 
lógica. Resulta prioritario mencionar que la mayoría de los estudios que intentan relacionar la depresión postictus con el deterioro cognitivo no utiliza baterías neuropsicológicas que permitan estudiar diferentes dominios cognitivos; en su lugar suelen utilizar instrumentos de cribado cognitivo, como el Minimental State Examination (MMSE). Estos tests de cribado no permiten establecer relaciones fiables entre el nivel cognitivo de la persona y su estado afectivo, ya que únicamente sirven como indicadores del estado cognitivo, no para la evaluación de déficits cognitivos específicos [34].

Otra limitación de los estudios es excluir a los pacientes con afasia, fatiga, malestar o estrés, por considerar que estos síntomas, a menudo presentes en la fase subaguda (4-30 días postictus), son un obstáculo para realizar la evaluación neuropsicológica de forma temprana [35]. Según estos autores, la incertidumbre sobre la viabilidad y la fiabilidad de la evaluación cognitiva en la fase subaguda ha influido a la hora de posponer dicha evaluación. Sin embargo, si tenemos en cuenta que desde el punto de vista clínico ayudaría conocer las funciones cognitivas afectadas tras el ictus para la toma de decisiones sobre qué estrategia de rehabilitación sería mejor para el paciente, resulta sorprendente que el estado cognitivo se haya omitido en la mayoría de los modelos de predicción de resultados en la etapa temprana tras el ictus.

Así pues, y teniendo en cuenta únicamente los estudios que han utilizado baterías de exploración neuropsicológicas para evaluar a estos pacientes, comentamos a continuación los hallazgos más recientes en esta cuestión, dado que la evidencia sobre la afectación cognitiva de los pacientes que han sufrido un AIT o ictus minor se remonta a la década de los ochenta.

Nys et al intentaron examinar la relación entre los síntomas depresivos y las funciones cognitivas en los pacientes con ictus. A pesar de que fueron excluidos los sujetos con AIT, los autores describieron el perfil de afectación neuropsicológica de los pacientes con depresión postictus (alteraciones visuoperceptivas de memoria y de lenguaje), que es diferente del de los pacientes con depresión sin historia de ictus, en los cuales predominan las dificultades de concentración, codificación de la información y velocidad de procesamiento de la información [34]. Un año más tarde, los mismos autores plantearon otro estudio en el que examinaron el valor predictivo del deterioro cognitivo en la fase aguda postictus como factor de riesgo a largo plazo para los síntomas depresivos y menor calidad de vida. En este segundo estudio concluyeron que el deterioro cognitivo temprano y los factores de riesgo vascular predicen la aparición de depresión postictus tardía y menor calidad de vida a los seis meses. Apuntaron incluso a que el tratamiento con antidepresivos ayuda a mejorar el rendimiento cognitivo en estos sujetos [36]. En esta misma línea de estudiar el papel de los déficits cognitivos postictus y la supervivencia a largo plazo, Oksala et al realizaron un estudio longitudinal a 12 años y demostraron la relación entre una menor supervivencia postictus y los déficits en las funciones ejecutivas, la memoria, las habilidades visuoespaciales y el lenguaje. El deterioro cognitivo global (medido con el MMSE) también se relacionaba con una menor supervivencia. Los déficits en las funciones ejecutivas y habilidades visuoespaciales permanecieron como predictores independientes después de controlar el efecto del deterioro cognitivo global y la gravedad del ictus [33]. Pendlebury et al compararon las características del perfil cognitivo entre pacientes que habían sufrido un ictus establecido y los que habían tenido un AIT. Concluyeron que el perfil cognitivo de los pacientes con AIT e ictus establecido es cualitativamente similar, aunque cuantitativamente los últimos tienen mayor afectación [37]. Asimismo, en los pacientes con ictus se ha demostrado la presencia de deterioro cognitivo tanto en la fase aguda como subaguda, con un patrón de disfunción en las funciones ejecutivas y en la memoria de trabajo. Según este estudio, los tests que miden estos dos dominios cognitivos fueron los mejores descriptores de la correlación entre cognición y depresión [38]. Además, este deterioro puede estar presente incluso en ictus leves e influir negativamente en la calidad de vida de los pacientes.

Otra cuestión de creciente interés es el efecto de la reserva cognitiva en la evolución de diferentes enfermedades. La hipótesis de la reserva cognitiva afirma que una mayor inteligencia y educación premórbida dotan al sujeto de la capacidad de reserva cerebral, es decir, de amortiguar o compensar los efectos del envejecimiento y la enfermedad. Recientemente, se ha demostrado que cuanto mayor es el nivel educativo (como referente de reserva cognitiva), menor es el deterioro cognitivo postictus. Además, se ha asociado con menor porcentaje de evolución a demencia, menor recurrencia de ictus y mejor supervivencia postictus [39].

Finalmente, en 2012 se publicó una revisión sobre la asociación entre depresión, deterioro cognitivo y disfunción ejecutiva postictus. Se incluyeron únicamente estudios que utilizaban baterías neuropsicológicas para evaluar el rendimiento cognitivo; 
en total se analizaron seis trabajos originales. Concluyeron que la depresión postictus se asocia con mayor deterioro cognitivo, y que los déficits cognitivos postictus más frecuentes son de funciones ejecutivas, memoria, lenguaje y velocidad de procesamiento de la información. Los resultados también apuntaron a la asociación entre la gravedad de la depresión y la magnitud de los déficits cognitivos, y éstos son hasta tres veces más frecuentes en los pacientes con depresión grave, comparados con los que presentan depresión leve [32].

\section{Consecuencias de la depresión postictus}

Muchos han sido los esfuerzos para estudiar las consecuencias de padecer esta enfermedad. El desarrollo de depresión postictus está vinculado con un peor pronóstico, y afecta negativamente a la mejora funcional de los pacientes, así como al proceso social y familiar de reintegración y supervivencia, y puede incrementar el riesgo de recurrencia vascular $[11,24,40]$. También se ha descrito que los pacientes con depresión postictus tienen de tres a cuatro veces más riesgo de mortalidad comparados con los pacientes no deprimidos [41]; incluso se ha descrito más riesgo de suicidio y una prevalencia de ideación suicida del 7-15\% [42-45]. Sin embargo, un estudio reciente concluyó que el perfil de sujetos con mayor riesgo de suicidio postictus no difería del perfil en la población general (sujetos con baja educación, menos ingresos y estado civil soltero). Encontraron que la pobre calidad de salud percibida y el bajo estado de ánimo a los tres meses postictus eran fuertes predictores de intentos de suicidio [46]. A pesar de las últimas aportaciones científicas, no está del todo claro si la depresión contribuye a la mortalidad postictus como un factor de riesgo individual o como un factor independiente que influye negativamente en otros factores de riesgo.

\section{Depresión postictus en ictus minor y ataque isquémico transitorio}

Pese a la vulnerabilidad de los pacientes que han sufrido un AIT o un ictus minor, existen muy pocos trabajos que incluyan o se centren en ellos. Como demuestra la nueva definición tisular de AIT de 2009 [47], la transitoriedad de los síntomas no excluye la presencia de lesiones isquémicas agudas que pueden llegarse a observar en uno de cada dos pacientes que cumplan la definición clásica de AIT (duración de los síntomas menor de 24 horas). Sna- phaan et al mantuvieron la hipótesis de que encontrarían una tasa de prevalencia de depresión postictus menor en estos sujetos y, sin embargo, no hallaron diferencias estadísticamente significativas al comparar la prevalencia de depresión postictus en el AIT respecto al ictus establecido [9]. Este hecho demuestra cómo esta complicación afectiva también está presente en los sujetos con clínica transitoria y que, por tanto, los estudios que en el futuro valoren la prevalencia de depresión postictus o los factores implicados en su desarrollo no pueden limitarse únicamente a los pacientes que hayan sufrido mayor deterioro neurológico [24]. De forma similar, Verbraak et al demostraron que a los seis meses los sujetos que habían sufrido un AIT tenían dificultades en las actividades de la vida diaria, percibían una calidad de vida más baja, y mostraban síntomas de ansiedad y depresión [48]. Recientemente, Moran et al hicieron una revisión sistemática para poner de manifiesto que los sujetos con AIT e ictus minor también sufren fatiga y alteraciones psicológicas y cognitivas postictus, a pesar de que no se les ofrece tratamiento para ello [49].

\section{Conclusiones}

Hasta la fecha existen numerosos estudios de depresión postictus, si bien los pacientes con ictus minor o AIT han quedado siempre al margen. Son sujetos de alto riesgo de recurrencia de ictus y, como demuestran diversos estudios recientes, también pueden desarrollar depresión postictus. En consecuencia, interesa conocer los factores predictores de desarrollo de depresión postictus presentes en los momentos iniciales tras el ictus minor o el AIT (fase aguda y subaguda), para poder detectar y predecir los pacientes con más riesgo de depresión postictus, y así establecer las estrategias terapéuticas más adecuadas. Para ello es imprescindible desarrollar nuevos estudios con mejor diseño que los actuales. Éstos deberían incluir el uso de diferentes escalas clínicas de depresión administradas en diferentes momentos temporales (estudios prospectivos y longitudinales) que apoyen el diagnóstico clínico realizado mediante entrevista de un profesional de la salud mental, también evaluaciones neuropsicológicas más completas que permitan establecer relaciones más fiables entre el deterioro cognitivo y el estado afectivo, así como biomarcadores de neuroimagen, y todo ello sin excluir en la selección de muestras a los pacientes con factores de riesgo para la depresión postictus ya descritos en la bibliografía. 
Bibliografía

1. Fernández de Bobadilla J, Sicras-Mainar A, Navarro-Artieda R, Planas-Comes A, Soto-Alvarez J, Sánchez-Maestre C, et al. Estimación de la prevalencia, incidencia, comorbilidades y costes directos asociados en pacientes que demandan atención por ictus en un ámbito poblacional español. Rev Neurol 2008; 46: 397-405.

2. The National Institute of Neurological Disorders and Stroke rt-PA Stroke Study Group. Tissue plasminogen activator for acute ischemic stroke. N Engl J Med 1995; 333: 1581-7.

3. Wahlgren N, Ahmed N, Dávalos A, Ford GA, Grond M, Hacke W, et al. Thrombolysis with alteplase for acute ischaemic stroke in the Safe Implementation of Thrombolysis in StrokeMonitoring Study (SITS-MOST): an observational study. Lancet 2007; 369: 275-82.

4. Balami JS, Sutherland BA, Edmunds LD, Grunwald IQ, Neuhaus AA, Hadley G, et al. A systematic review and meta-analysis of randomized controlled trials of endovascular thrombectomy compared with best medical treatment for acute ischemic stroke. Int J Stroke 2015; 10: 1168-78.

5. Jovin TG, Chamorro A, Cobo E, De Miquel MA, Molina CA Rovira A, et al. Thrombectomy within 8 hours after symptom onset in ischemic stroke. N Engl J Med 2015; 372: 2296-306.

6. Hackett ML, Yapa C, Parag V, Anderson CS. Frequency of depression after stroke: a systematic review of observational studies. Stroke 2005; 36: 1330-40.

7. Williams LS. Depression and stroke: cause or consequence? Semin Neurol 2005; 25: 396-409.

8. Carod-Artal FJ. Depresión postictus (I). Epidemiología, criterios diagnósticos y factores de riesgo. Rev Neurol 2006; 42: 169-75.

9. Snaphaan L, Van Der Werf S, Kanselaar K, De Leeuw FE. Post-stroke depressive symptoms are associated with poststroke characteristics. Cerebrovasc Dis 2009; 28: 551-7.

10. Paolucci S, Gandolfo C, Provinciali L, Torta R, Toso V. The Italian multicenter observational study on post-stroke depression (DESTRO). J Neurol 2006; 253: 556-62.

11. Espárrago-Llorca G, Castilla-Guerra L, Fernández-Moreno MC Ruiz-Doblado S, Jiménez-Hernández MD. Depresión postictus: una actualización. Neurologia 2015; 30: 23-31.

12. Tang WK, Chen YK, Liang HJ, Chu WCW, Mok VCT, Ungvari GS, et al. Location of infarcts and apathy in ischemic stroke. Cerebrovasc Dis 2013; 35: 566-71.

13. Jorge RE, Starkstein SE, Robinson RG. Apathy following stroke. Can J Psychiatry 2010; 55: 350-4.

14. Caeiro L, Ferro JM, Figueira ML. Apathy in acute stroke patients. Eur J Neurol 2012; 19: 291-7.

15. Carod-Artal FJ. Depresión postictus (II). Diagnóstico diferencial, complicaciones y tratamiento. Rev Neurol 2006; 42: 238-44.

16. Appelros P, Viitanen M. Prevalence and predictors of depression at one year in a Swedish population-based cohort with first-ever stroke. J Stroke Cerebrovasc Dis 2004; 13: 52-7.

17. Van Dalen JW, Van Charante EPM, Nederkoorn PJ, Van Gool W, Richard E. Poststroke apathy. Stroke 2013; 44: 851-60.

18. Sagen U, Vik TG, Moum T, Mørland T, Finset A, Dammen T. Screening for anxiety and depression after stroke: comparison of the Hospital Anxiety and Depression Scale and the Montgomery and Åsberg Depression Rating Scale. J Psychosom Res 2009; 67: 325-32.

19. Kang HJ, Stewart R, Kim JM, Jang JE, Kim SY, Bae KY, et al. Comparative validity of depression assessment scales for screening poststroke depression. J Affect Disord 2013; 147: 186-91.

20. Aben I, Lodder J, Honig A, Lousberg R, Boreas A, Verhey F. Focal or generalized vascular brain damage and vulnerability to depression after stroke: a 1-year prospective follow-up study. Int Psychogeriatr 2006; 18: 19-35.

21. Aben I, Verhey F, Lousberg R, Lodder J, Honig A. Validity of the Beck Depression Inventory, Hospital Anxiety and Depression Scale, SCL-90, and Hamilton Depression Rating Scale as screening instruments for depression in stroke patients. Psychosomatics 2002; 43: 386-93.

22. Turner A, Hambridge J, White J, Carter G, Clover K, Nelson L, et al. Depression screening in stroke: a comparison of alternative measures with the structured diagnostic interview for the diagnostic and statistical manual of mental disorders, fourth edition (major depressive episode) as criterion standard. Stroke 2012; 43: 1000-5.

23. Meader N, Moe-Byrne T, Llewellyn A, Mitchell AJ. Screening for poststroke major depression: a meta-analysis of diagnostic validity studies. J Neurol Neurosurg Psychiatry 2014; 85: 198-206.

24. Altieri M, Maestrini I, Mercurio A, Troisi P, Sgarlata E, Rea V et al. Depression after minor stroke: prevalence and predictors. Eur J Neurol 2012; 19: 517-21.

25. Purroy F, Jiménez-Caballero PE, Gorospe A, Torres MJ, Álvarez-Sabín J, Santamarina E, et al. Recurrent transient ischaemic attack and early risk of stroke: data from the PROMAPA study. J Neurol Neurosurg Psychiatry 2013; 84: 596-603.

26. Purroy-García F, Montaner J, Delgado P, Ribó M, ArenillasLara JF, Quintana M, et al. Factores clínicos predictores de la aparición de episodios vasculares tras un ataque isquémico transitorio. Rev Neurol 2004; 38: 416-21.

27. Alexopoulos G, Meyers B, Young R, Campbell S, Silbersweig D, Charlson M. 'Vascular depression' hypothesis. Arch Gen Psychiatry 1997; 54: 915-22.

28. Lockwood K, Alexopoulos G, Van Gorp W. Executive dysfunction in geriatric depression. Am J Psychiatry 2002; 159: 1119-26.

29. Alexopoulos GS. The depression-executive dysfunction syndrome of late life. Am J Geriatr Psychiatry 2001; 9: 22-9.

30. American Psychiatric Association. Diagnostic and statistical manual of mental disorders, fifth edition. Arlington, VA: American Psychiatric Publishing; 2013.

31. De Ryck A, Brouns R, Geurden M, Elseviers M, De Deyn PP, Engelborghs S. Risk factors for poststroke depression: identification of inconsistencies based on a systematic review. J Geriatr Psychiatry Neurol 2014; 27: 147-58.

32. Terroni L, Sobreiro MFM, Conforto AB, Adda CC, Guajardo VD, De Lucia MCS, et al. Association among depression, cognitive impairment and executive dysfunction after stroke. Dement Neuropsychol 2012; 6: 152-7.

33. Oksala NKJ, Jokinen H, Melkas S, Oksala A, Pohjasvaara T, Hietanen M, et al. Cognitive impairment predicts poststroke death in long-term follow-up. J Neurol Neurosurg Psychiatry 2009; 80: 1230-5.

34. Nys GMS, Van Zandvoort MJE, Van der Worp HB, De Haan EHF, De Kort PLM, Kappelle LJ. Early depressive symptoms after stroke: neuropsychological correlates and lesion characteristics. J Neurol Sci 2005; 228: 27-33.

35. Van Zandvoort MJE, Kessels RPC, Nys GMS, De Haan EHF, Kappelle LJ. Early neuropsychological evaluation in patients with ischaemic stroke provides valid information. Clin Neurol Neurosurg 2005; 107: 385-92.

36. Nys GMS, Van Zandvoort MJE, Van der Worp HB, De Haan EHF, De Kort PLM, Jansen BPW, et al. Early cognitive impairment predicts long-term depressive symptoms and quality of life after stroke. J Neurol Sci 2006; 247: 149-56.

37. Pendlebury ST, Wadling S, Silver LE, Mehta Z, Rothwell PM. Transient cognitive impairment in TIA and minor stroke. Stroke 2011; 42: 3116-21.

38. Hommel M, Carey L, Jaillard A. Depression: cognition relations after stroke. Int J Stroke 2013; 1-4.

39. Ojala-Oksala J, Jokinen H, Kopsi V, Lehtonen K, Luukkonen L, Paukkunen A, et al. Educational history is an independent predictor of cognitive deficits and long-term survival in postacute patients with mild to moderate ischemic stroke. Stroke 2012; 43: 2931-5.

40. Gilsanz P, Walter S, Tchetgen EJT, Patton KK, Moon JR, Capistrant BD, et al. Changes in depressive symptoms and incidence of first stroke among middle-aged and older US adults. J Am Hear Assoc 2015; 4: e001923.

41. Hadidi N, Treat-Jacobson DJ, Lindquist R. Poststroke depression and functional outcome: a critical review of literature. Heart Lung 2009; 38: 151-62.

42. Tang WK, Caeiro L, Lau CG, Liang H, Mok V, Ungvari GS, et al. Apathy and suicide-related ideation 3 months after stroke: a cross-sectional study. BMC Neurol 2015; 15: 1-5. 
43. Pompili M, Venturini P, Campi S, Seretti ME, Montebovi F, Lamis DA, et al. Do stroke patients have an increased risk of developing suicidal ideation or dying by suicide? An overview of the current literature. CNS Neurosci Ther 2012; 18: 711-21.

44. Pohjasvaara T, Vataja R, Leppävuori A, Kaste M, Erkinjuntti T. Depression is an independent predictor of poor long-term functional outcome post-stroke. Eur J Neurol 2001; 8: 315-9.

45. Pohjasvaara T, Vataja R, Leppävuori A, Kaste M, Erkinjuntti T. Suicidal ideas in stroke patients 3 and 15 months after stroke. Cerebrovasc Dis 2001; 12: 21-6.

46. Eriksson M, Glader E, Norrving B, Asplund K. Poststroke suicide attempts and completed suicides. Neurology 2015; 84: 1732-8.

47. Easton JD, Saver JL, Albers GW, Alberts MJ, Chaturvedi S, Feldmann E, et al. Definition and evaluation of transient ischemic attack: a scientific statement for healthcare professionals from the American Heart Association/American Stroke Association Stroke Council. Stroke 2009; 40: 2276-93.

48. Verbraak ME, Hoeksma AF, Lindeboom R, Kwa VIH. Subtle problems in activities of daily living after a transient ischemic attack or an apparently fully recovered non-disabling stroke. J Stroke Cerebrovasc Dis 2012; 21: 124-30.

49. Moran GM, Fletcher B, Calvert M, Feltham MG, Sackley C, Marshall T. A systematic review investigating fatigue, psychological and cognitive impairment following TIA and minor stroke: protocol paper. Syst Rev 2013; 2: 72.

50. Berg A, Palomäki H, Lehtihalmes M, Lönnqvist JJ, Kaste M. Poststroke depression: an 18-month follow-up. Stroke 2003; 34: 138-43.

51. Hackett ML, Anderson CS. Predictors of depression after stroke: a systematic review of observational studies. Stroke 2005; 36: 2296-301.

52. De Ryck A, Brouns R, Fransen E, Geurden M, Van Gestel G Wilssens I, et al. A prospective study on the prevalence and risk factors of poststroke depression. Cerebrovasc Dis Extra 2013; 3: 1-13.
53. Masskulpan P, Riewthong K, Dajpratham P, Kuptniratsaikul V. Anxiety and depressive symptoms after stroke in 9 rehabilitation centers. J Med Assoc Thail 2008; 91: 1595-602.

54. Suenkeler IH, Nowak M, Misselwitz B, Kugler C, Schreiber W, Oertel WH, et al. Timecourse of health-related quality of life as determined 3, 6 and 12 months after stroke. Relationship to neurological deficit, disability and depression. J Neurol 2002; 249: 1160-7.

55. Carson AJ, MacHale S, Allen K, Lawrie SM, Dennis M, House A, et al. Depression after stroke and lesion location: a systematic review. Lancet 2000; 356: 122-6.

56. Yang S, Hua P, Shang X, Cui Z, Zhong S, Gong G, et al. A significant risk factor for poststroke depression: the depressionrelated subnetwork. J Psychiatry Neurosci 2015; 14: 1-10.

57. Tang WK, Chen Y, Liang H, Chu WCW, Mok VCT, Ungvari GS, et al. Cerebral microbleeds as a predictor of 1-year outcome of poststroke depression. Stroke 2014; 45: 77-81.

58. Narushima K, Kosier JT, Robinson RG. A reappraisal of poststroke depression, intra- and inter-hemispheric lesion location using meta-analysis. J Neuropsychiatry Clin Neurosci 2003; 15: 422-30.

59. Bamford J, Sandercock P, Dennis M, Burn J, Warlow C. Classification and natural history of clinically identifiable subtypes of cerebral infarction. Lancet 1991; 337: 1521-6.

60. Provinciali L, Paolucci S, Torta R, Toso V, Gobbi B, Gandolfo C. Depression after first-ever ischemic stroke: the prognostic role of neuroanatomic subtypes in clinical practice. Cerebrovasc Dis 2008; 26: 592-9.

61. Camões-Barbosa A, Sequeira-Medeiros L, Duarte N, Meneses C. Factores preditivos de depressão pós-acidente vascular cerebral estudo retrospectivo numa unidade de reabilitação. Acta Med Port 2011; 24: 175-85.

62. Fuentes B, Ortiz X, Sanjose B, Frank A, Díez-Tejedor E. Post-stroke depression: can we predict its development from the acute stroke phase? Acta Neurol Scand 2009; 120: 150-6.

\section{Update on post-stroke depression: posing new challenges in patients with a minor stroke or transient ischaemic attack}

Introduction. Stroke is a leading cause of disability in adults. The development of depressive symptoms is the most common emotional complication. To date, most studies of post-stroke depression have excluded patients who have suffered a minor stroke or transient ischaemic attack (TIA), although they are equally vulnerable subgroups of this sickness.

Aim. We present a review of published studies of post-stroke depression to elucidate aspects that have already been widely demonstrated and those who need more evidence.

Development. The post-stroke depression is both frequent in patients with established stroke and minor stroke or TIA. Although there are discrepancies in the definition used, in up to one out of three patients will develop this complication. We have identified risk factors of post-stroke depression with a broad scientific background (female, history of depression or other psychiatric disorders, stroke severity, functional impairment) and other without it (quality of life, cognitive impairment and neuroimaging biomarkers). The main methodological limitations found are: confusion between poststroke depression and depressive symptoms; variability in rating scales used; and temporal variability in the time of the evaluation of mood. To date very few studies focused on minor stroke or TIA.

Conclusions. Further studies are required with improved design in order to help establish the risk of post-stroke depression at different times after the stroke, minor stroke or TIA and the importance of all the factors described above.

Key words. Minor stroke. Neuropsychology. Post-stroke depression. Prognosis. Review. Transient ischaemic attack. 